\title{
Softening the Robustness of Optimization Problems: A New Budgeted Uncertainty Approach
}

\author{
Romain Guillaume ${ }^{1}$, Adam Kasperski ${ }^{2}\left[\right.$, and Paweł Zieliński ${ }^{2(\bowtie)}(\mathbb{C}$ \\ 1 Université de Toulouse-IRIT, Toulouse, France \\ Romain.Guillaume@irit.fr \\ 2 Wrocław University of Science and Technology, Wrocław, Poland \\ \{Adam.Kasperski, Pawel.Zielinski\}@pwr.edu.pl
}

\begin{abstract}
In this paper an optimization problem with uncertain parameters is discussed. In the traditional robust approach a pessimistic point of view is assumed. Namely, a solution is computed under the worst possible parameter realizations, which can lead to large deterioration of the objective function value. In this paper a new approach is proposed, which assumes a less pessimistic point of view. The complexity of the resulting problem is explored and some methods of solving its special cases are presented.
\end{abstract}

Keywords: Robustness $\cdot$ Uncertainty $\cdot$ Optimization

\section{Introduction}

In this paper we wish to investigate the following optimization problem with uncertain parameters:

$$
\begin{array}{ll}
\max (\min ) & \boldsymbol{c}^{T} \boldsymbol{x}, \\
\text { s.t. } & \widetilde{\boldsymbol{a}}_{i}^{T} \boldsymbol{x} \leq b_{i} \quad i \in[m], \\
& \boldsymbol{x} \in \mathbb{X} \subseteq \mathbb{R}_{+}^{n} .
\end{array}
$$

In formulation (1), $\boldsymbol{x}$ is $n$-vector of nonnegative decision variables, $\boldsymbol{c}$ is $n$-vector of deterministic objective function coefficients, $\widetilde{\boldsymbol{a}}_{i}=\left(\widetilde{a}_{i 1}, \ldots, \widetilde{a}_{i n}\right)$ is $n$-vector of uncertain constraint coefficients, $i \in[m]([m]$ denotes the set $\{1, \ldots, m\})$, and $\mathbb{X}$ is a bounded subset of $\mathbb{R}_{+}^{n}$, where $\mathbb{R}_{+}$is the set of nonnegative reals. For example, if $\mathbb{X}$ is a bounded polyhedron, then (1) is an uncertain linear programming problem. If $\mathbb{X} \subseteq\{0,1\}^{n}$, then (1) is an uncertain combinatorial optimization problem. We will first assume that the right-hand sides $b_{i}, i \in[\mathrm{m}]$, of the constraints are deterministic. Later, we will also discuss the case with uncertain $b_{i}$. We can assume w.l.o.g. that the objective function coefficients are

Romain Guillaume was partially supported by the project caasc ANR-18-CE10-0012 of the French National Agency for Research. Adam Kasperski and Paweł Zieliński were supported by the National Science Centre, Poland, grant 2017/25/B/ST6/00486.

(c) Springer Nature Switzerland AG 2020

M.-J. Lesot et al. (Eds.): IPMU 2020, CCIS 1237, pp. 187-200, 2020.

https://doi.org/10.1007/978-3-030-50146-4_15 
precise. Otherwise, in a minimization problem, we can replace the objective function with minimization of a new variable $t$ and add one additional uncertain constraint $\widetilde{\boldsymbol{c}}^{T} \boldsymbol{x}-t \leq 0$. The transformation for maximization problems is similar.

A method of solving (1) depends on the information available. If $\widetilde{\boldsymbol{a}}_{i}$ is a vector of random variables with known probability distribution, then the $i$ th imprecise constraint can be replaced with a chance constraint of the form $\operatorname{Pr}\left(\widetilde{\boldsymbol{a}}_{i}^{T} \boldsymbol{x} \leq b_{i}\right) \geq$ $1-\epsilon$, where $\epsilon \in[0,1)$ is a given risk level [8]. Assume that we only know that $\widetilde{\boldsymbol{a}}_{i} \in \mathcal{U}_{i} \subseteq \mathbb{R}^{n}$, where $\mathcal{U}_{i}$ is a given uncertainty (scenario) set. In this paper we use the following interval model of uncertainty [3]. For each uncertain coefficient $\widetilde{a}_{i j}$ an interval $\left[\widehat{a}_{i j}-\Delta_{i j}, \widehat{a}_{i j}+\Delta_{i j}\right]$ is provided, where $\widehat{a}_{i j}$ is the nominal value of $\widetilde{a}_{i j}$ and $\Delta_{i j}$ is the maximal deviation of the value of $\widetilde{a}_{i j}$ from its nominal one. The interval can be interpreted as a support of random variable $\widetilde{a}_{i j}$, symmetrically distributed around its nominal (expected) value [3]. Set $\mathcal{U}_{i}$ is the Cartesian product of the uncertainty intervals $\left[\widehat{a}_{i j}-\Delta_{i j}, \widehat{a}_{i j}+\Delta_{i j}\right], j \in[n]$. Let $\widehat{\boldsymbol{a}}_{i} \in \mathbb{R}^{n}$ be a vector of the nominal constraint coefficients. After replacing the uncertain vectors $\widetilde{\boldsymbol{a}}_{i}$ with their nominal counterparts $\widehat{\boldsymbol{a}}_{i}$ for each $i \in[\mathrm{m}]$, we get a deterministic nominal problem with the optimal objective value equal to $\widehat{c}$. Using the robust optimization framework $[2,10,13]$, the $i$ th imprecise constraint can be replaced with

$$
\max _{\boldsymbol{a}_{i} \in \mathcal{U}_{i}} \boldsymbol{a}_{i}^{T} \boldsymbol{x} \leq b_{i}
$$

which ensures that $\boldsymbol{x}$ is feasible for all scenarios $\boldsymbol{a}_{i} \in \mathcal{U}_{i}$.

The application of strict robustness concept (2) results in a very conservative constraint, in which we assume by the non-negativity of $\boldsymbol{x}$, that the true realization of all the coefficients will be at $\widehat{a}_{i j}+\Delta_{i j}, j \in[n]$. Hence the objective value of the strict robust solution can be much less than $\widehat{c}$. This phenomenon is called a price of robustness [3] and large price of robustness is often regarded as the main drawback of the strict robust optimization. However, in many practical situations, the true realization of $\widetilde{\boldsymbol{a}}_{i}$ will be rather closer to $\widehat{\boldsymbol{a}}_{i}$, as the extreme values of the coefficients are less probable to occur, especially when everything goes smoothly without any perturbations [5].

Several approaches have been proposed in the literature to soften the strict robustness. One of the most popular was introduced in [3]. The key idea is to assume that at most $\Gamma_{i}$ coefficients in the $i$ th constraint will take the values different than their nominal ones. To simplify presentation, we will assume that $\Gamma_{i}$ is an integer in $\{0, \ldots, n\}$. Accordingly, the $i$ th constraint becomes then

$$
\max _{\left\{\boldsymbol{a}_{i} \in \mathcal{U}_{i}:\left|\left\{a_{i j}: a_{i j} \neq \widehat{a}_{i j}, j \in[n]\right\}\right| \leq \Gamma_{i}\right\}} \boldsymbol{a}_{i}^{T} \boldsymbol{x} \leq b_{i} .
$$

Notice that the case when $\Gamma_{i}=0$ only ensures that $x$ is feasible under the nominal scenario (we get the nominal problem). On the other hand, $\Gamma_{i}=n$ ensures that $\boldsymbol{x}$ is feasible under all scenarios and, in this case, (3) is equivalent to (2). The parameter $\Gamma_{i}$ allows decision makers to control the robustness of the constraint. By changing $\Gamma_{i}$, we get a family of solutions with different levels of robustness. However, it is still assumed that $\Gamma_{i}$ constraint coefficients may take their worst values, which represents a pessimistic point of view. In $[5,12]$ 
an approach to soften the robustness of (2) was proposed. The idea is to assume that the solution cost should be of some predefined distance to $\widehat{c}$, which can be achieved by allowing additional constraint violations (see [12] for details). Another method consists in replacing very conservative minmax criterion with the minmax regret one (see $[9,10]$ for more details and the references given there). However, the minmax regret problems are typically hard to solve, even for linear programming problems [1].

In this paper we propose a new approach to soften the strict robustness. The idea will be to modify the approach proposed in [3], by replacing the pessimistic point of view with a more optimistic one. We will still assume that $\Gamma_{i}$ constraint coefficients can take the worst values. However, contrary to (3), we will assume that this will happen in the best possible case. Consequently, the objective function will be optimized over the larger set of feasible solutions and the optimal objective value will be closer to $\widehat{c}$.

\section{New Concept of Choosing Robust Solutions}

In this section we propose a new concept to soften the conservatism of the strict robust approach. We will use the same model of uncertainty as the one described in the previous section. Namely, for each uncertain coefficient $\tilde{a}_{i j}$ we define the uncertainty interval $\left[\widehat{a}_{i j}-\Delta_{i j}, \widehat{a}_{i j}+\Delta_{i j}\right]$. Also $\Gamma_{i} \in\{0, \ldots, n\}$ specifies the number of coefficients in the $i$ th constraint, whose values can be different from their nominal ones. Let $\Phi_{i}=\left\{\boldsymbol{\delta}_{i} \in\{0,1\}^{n}: \sum_{j \in[n]} \delta_{i j}=\Gamma_{i}\right\}$. A fixed vector $\boldsymbol{\delta}_{i} \in \Phi_{i}$ induces the following convex uncertainty set:

$$
\mathcal{U}_{\boldsymbol{\delta}_{i}}=\left\{\boldsymbol{a}_{i} \in \mathbb{R}^{n}: a_{i j} \in\left[\widehat{a}_{i j}+\delta_{i j} \Delta_{i j}\right], j \in[n]\right\} .
$$

Since $\boldsymbol{x} \in \mathbb{R}_{+}^{n}$, the constraint (3) can be rewritten equivalently as

$$
\max _{\boldsymbol{\delta}_{i} \in \Phi_{i}} \max _{\boldsymbol{a}_{i} \in \mathcal{U}_{\boldsymbol{\delta}_{i}}} \boldsymbol{a}_{i}^{T} \boldsymbol{x} \leq b_{i} .
$$

We can now provide the following interpretation of (4). Given a solution $\boldsymbol{x}$, we first choose the worst uncertainty set $\mathcal{U}_{\boldsymbol{\delta}_{i}}$ and then the worst scenario $\boldsymbol{a}_{i}$ in this set. This represents a pessimistic point of view. From an optimistic point of view, we can assume that the best uncertainty set is chosen in the first step, which leads to the following constraint:

$$
\min _{\boldsymbol{\delta}_{i} \in \Phi_{i} \max _{i} \in \mathcal{U}_{\boldsymbol{\delta}_{i}}} \boldsymbol{a}_{i}^{T} \boldsymbol{x} \leq b_{i}
$$

If $\mathbb{X}$ is a polyhedron, then the set of feasible solutions to (4) is convex, because it can be represented by an intersection of polyhedral sets. Indeed, $\max _{\boldsymbol{a}_{i} \in \mathcal{U}_{\boldsymbol{\delta}_{i}}} \boldsymbol{a}_{i}^{T} \boldsymbol{x}=\sum_{j \in[n]}\left(\widehat{a}_{i j}+\delta_{i j} \Delta_{i j}\right) x_{j}$ and (4) is equivalent to the family (conjunction) of the linear constraints $\sum_{j \in[n]}\left(\widehat{a}_{i j}+\delta_{i j} \Delta_{i j}\right) x_{j} \leq b_{i}$ for all $\boldsymbol{\delta}_{i} \in \Phi_{\boldsymbol{\delta}_{i}}$. On the other hand, the set of feasible solutions to (5) need not to be convex, because it is a union of polyhedral sets. Constraint (5) is equivalent to 


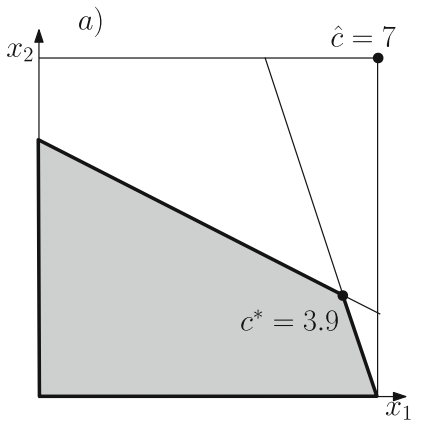

$\max 3 x_{1}+4 x_{2}$

s.t. $\tilde{a}_{1} x_{1}+\tilde{a}_{2} x_{2} \leq 3$ $x_{1}, x_{2} \in[0,1]$

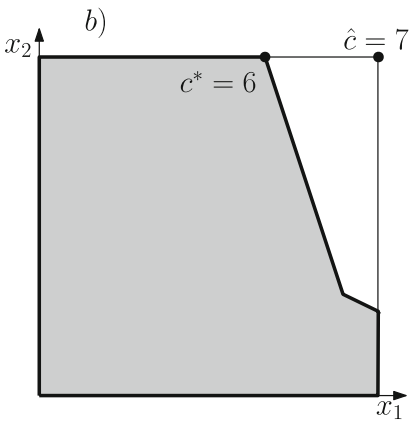

$\tilde{a}_{1}=[\hat{2}-1, \hat{2}+1]$

$\tilde{a}_{2}=[\hat{1}-3, \hat{1}+3]$

Fig. 1. A sample problem with one uncertain constraint and $\Gamma_{1}=1$. In a) the set of feasible solutions using (4) and in b) the set of feasible solutions using (5) are shown.

$\sum_{j \in[n]}\left(\widehat{a}_{i j}+\delta_{i j} \Delta_{i j}\right) x_{j} \leq b_{i}$ for at least one $\boldsymbol{\delta}_{i} \in \Phi_{\boldsymbol{\delta}_{i}}$, so it is a disjunction of a family of linear constraints.

Let us illustrate this by the example shown in Fig. 1. The set of feasible solutions to the nominal problem with $\tilde{a}_{1}=2$ and $\tilde{a}_{2}=1$ is $[0,1] \times[0,1]$, which gives the optimal solution $x_{1}=x_{2}=1$ with $\widehat{c}=7$. Using the concept (4) we get the set of feasible solutions shown in Fig. 1a, which is conjunction of the constraints $3 x_{1}+x_{2} \leq 3$ and $2 x_{1}+4 x_{2} \leq 3$. The optimal solution is $x_{1}=0.9$, $x_{2}=0.3$ with the optimal objective value equal 3.9 . Using the concept (5) we get the set of feasible solutions depicted in Fig. 1b, which is disjunction of the constraints $3 x_{1}+x_{2} \leq 3$ and $2 x_{1}+4 x_{2} \leq 3$. The optimal solution is then $x_{1}=0.67, x_{2}=1$ with the objective value equal to 6 . This solution has lower price of robustness. Observe, however, that the resulting set of feasible solutions is not convex.

\section{Solving the Problem}

Using the concept (5) we can rewrite the uncertain problem (1) as follows:

$$
\begin{aligned}
& \max \boldsymbol{c}^{T} \boldsymbol{x} \\
& \text { s.t. } \sum_{j \in[n]}\left(\widehat{a}_{i j}+\delta_{i j} \Delta_{i j}\right) x_{j} \leq b_{i} i \in[m], \\
& \sum_{j \in[n]} \delta_{i j}=\Gamma_{i} \quad i \in[m], \\
& \delta_{i j} \in\{0,1\} \quad i \in[m], j \in[n], \\
& x \in \mathbb{X} \text {. }
\end{aligned}
$$

Binary variables $\delta_{i j}$ select the uncertainty set $\mathcal{U}_{\delta_{i}}$ in the $i$ th constraint. The nonlinear terms $\delta_{i j} x_{j}$ can be linearized by applying standard techniques. In 
consequence, if $\mathbb{X}$ is described by a system of linear constraints, then the resulting problem is a mixed integer linear one. In Sect. 5 we will investigate the complexity of (6) and two its special cases.

\section{Illustrative Example}

In this section we will evaluate our concept by computational experiments. We will perform experiments for the continuous 0-1 knapsack problem with uncertain constraint coefficients (weights). The following model is the counterpart of (6) for the uncertain continuous 0-1 knapsack problem:

$$
\begin{array}{ll}
\max & \boldsymbol{c}^{T} \boldsymbol{x} \\
& \sum_{j \in[n]}\left(\widehat{a}_{j}+\delta_{j} \Delta_{j}\right) x_{j} \leq b, \\
& \sum_{j \in[n]} \delta_{j}=\Gamma, \\
& \boldsymbol{\delta} \in\{0,1\}^{n}, \\
& \boldsymbol{x} \in[0,1]^{n} .
\end{array}
$$

An instance of the problem is generated as follows. We fix $n=100, c_{j}$ is a random integer, uniformly distributed in $[10,100], \widehat{a}_{j}$ is a random integer, uniformly distributed in $[20,60]$ and $\Delta_{j}=\sigma \widehat{a}_{j}$, where $\sigma$ is a random real from the interval $[0,1]$. We also fix $b=0.4 \sum_{j \in[n]} \widehat{a}_{i}$. By changing $\Gamma$ from 0 to 100, we obtained a family of solutions to the pessimistic problem with the constraint (4) and to the optimistic problem with the constraint (5), i.e. to problem (7). Let $\boldsymbol{x}$ be a feasible solution for some fixed $\Gamma$. We define $\operatorname{dev}(\boldsymbol{x})=\left(\widehat{c}-\boldsymbol{c}^{T} \boldsymbol{x}\right) / \widehat{c}$, which is the price of robustness of $\boldsymbol{x}$, expressing a relative distance of $\boldsymbol{c}^{T} \boldsymbol{x}$ to the optimal objective function value of the nominal problem. The quantity $\operatorname{viol}(\boldsymbol{x})$ is an empirical estimation of the probability of the constraint violation, which is computed as follows. We generated 10000 random scenarios (constraint coefficient values) by choosing uniformly at random the value of $\tilde{a}_{j}$ from $\left[\widehat{a}_{j}-\Delta_{j}, \widehat{a}_{j}+\Delta_{j}\right]$, $j \in[n]$. Then $\operatorname{viol}(\boldsymbol{x})$ is the fraction of scenarios under which $\boldsymbol{x}$ is infeasible.

The obtained results are shown in Fig. 2. As one can expect, the optimistic approach results in lower price of robustness, but also in larger risk of the constraint violation. Both approaches are equivalent for the boundary values of $\Gamma$ equal to 0 or 100 . Furthermore, the pessimistic problem quickly decreases the constraint violation $\operatorname{viol}(\boldsymbol{x})$ and increases the price of robustness $\operatorname{dev}(\boldsymbol{x})$ as $\Gamma$ increases and a compromise, between $\operatorname{viol}(\boldsymbol{x})$ and $\operatorname{dev}(\boldsymbol{x})$, is reached for $\Gamma \approx 10$. While for the optimistic problem a similar compromise is reached for $\Gamma \approx 75$. Accordingly, combining the two approaches, i.e. the pessimistic and optimistic points of view, one can provide a larger family of solutions whose profile is shown in Fig. 2. One of them can be ultimately chosen by the decision maker, who can take a risk-aversion or some other factors into account. 

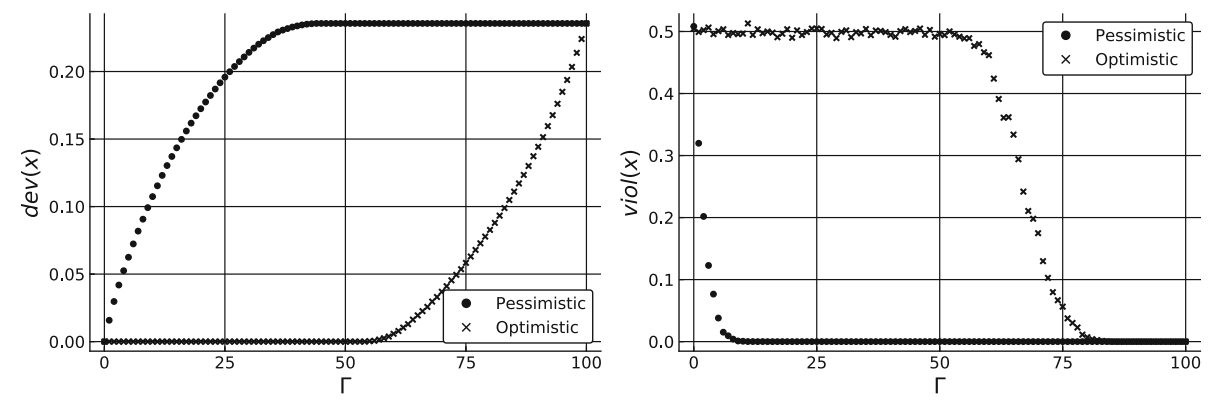

Fig. 2. The values of $\operatorname{dev}(\boldsymbol{x})$ and $\operatorname{viol}(\boldsymbol{x})$, where $\boldsymbol{x}$ is an optimal solution to the pessimistic (4) or optimistic (5) problem for $\Gamma \in\{0, \ldots, 100\}$.

\section{Uncertain Constraint Coefficients}

In this section we proceed with the study of problem (6). We provide a negative complexity result for it and some positive results for its two special cases. The following theorem characterizes the complexity of problem (6):

Theorem 1. Problem (6) is strongly NP-hard and not at all approximable even if $\mathbb{X}=[0,1]^{n}$ and $\Gamma_{i} \in\{0,1\}$ for each $i \in[m]$.

Proof. Consider the strongly NP-complete 3-SAT problem [6], in which we are given a set of boolean variables $\left\{x_{1}, \ldots, x_{n}\right\}$ and a set of clauses $C_{1}, \ldots, C_{m}$. Each clause $C_{i}$ contains three literals $\left\{p_{i}, q_{i}, r_{i}\right\}$, where $p_{i}, q_{i}, r_{i} \in\left\{x_{1}, \bar{x}_{1}, \ldots, x_{n}, \bar{x}_{n}\right\}$. We ask if there is a $0-1$ assignment to the variables which satisfies all the clauses. Given an instance of 3-SAT we build the following program:

$$
\begin{aligned}
& \max (1-t) \\
& \text { s.t. }\left(-1+2 \delta_{1 j}\right) x_{j}+\left(-1+2 \delta_{2 j}\right) \bar{x}_{j} \leq-1 j \in[n] \text {, } \\
& \delta_{1 j}+\delta_{2 j}=1 \quad j \in[n] \text {, } \\
& p_{i}+q_{i}+r_{i} \geq 1-t \quad \forall C_{i}=\left\{p_{i}, q_{i}, r_{i}\right\} \text {, } \\
& \delta_{1 j}, \delta_{2 j} \in\{0,1\} \quad j \in[n], \\
& x_{j}, \bar{x}_{j}, t \in[0,1] \quad j \in[n] .
\end{aligned}
$$

Observe that (8) is a special case of (6), where $\Gamma_{i} \in\{0,1\}$ for each constraint and $\mathbb{X}=[0,1]^{n+1}$. Notice that the clause constrains can be equivalently rewritten as $-p_{i}-q_{i}-r_{i}-t \leq-1$ with $\Gamma_{i}=0$. In any feasible solution to (8), we must have $x_{j}=1, \bar{x}_{j}=0$ or $x_{j}=1, \bar{x}_{j}=0$ for each $j \in[n]$. Indeed, the constraint $\delta_{1 j}+\delta_{2 j}=1$ forces $x_{j}-\bar{x}_{j} \leq-1$ or $\bar{x}_{j}-x_{j} \leq-1$. Since $x_{j}, \bar{x}_{j} \in[0,1]$ the property is true. Also, (8) is feasible, because by setting $t=1$, we can satisfy all the constraints associated with the clauses. We will show that the answer to 3 -SAT is yes if the optimal objective value to (8) is 1 and 0 , if the answer is no.

Assume that the answer to 3 -SAT is yes. Then, there is a $0-1$ assignment to the variables $x_{1}, \ldots, x_{n}$, which satisfies all the clauses. We construct a feasible solution to (8) as follows. The values of $x_{j}$ are the same as in the truth assignment 
and $\bar{x}_{j}=1-x_{j}, j \in[n]$. Also, $\delta_{1 j}=1-x_{j}$ and $\delta_{2 j}=1-\bar{x}_{j}$ for each $j \in[n]$. Finally $t=0$. The clause constraints are satisfied by the assumption that $x_{1}, \ldots, x_{n}$ satisfies all the clauses. The objective value for this feasible solution equals 1 .

Assume that the answer to 3-SAT is no, but the optimal objective value to (8) is greater than 0 , so $t<1$. Since $x_{j}, \bar{x}_{j} \in\{0,1\}$ in every feasible solution to (8), there must be at least one variable with the value of 1 in each clause constraint. But, as the answer to 3 -SAT is no, there must be $j$ such that $x_{j}=\bar{x}_{j}$, which contradicts the feasibility of $x_{j}$ and $\bar{x}_{j}$ for $t<1$. Hence $t=1$ and the optimal objective value of $(8)$ is 0 .

\section{$5.1 \quad$ 0-1 Knapsack Problem}

In this section we study the 0-1 knapsack problem with uncertain weights that is a special case of problem (6) in which $\mathbb{X}=\{0,1\}^{n}$ and $m=1$, i.e. we investigate the following problem:

$$
\begin{array}{lll}
\max & \sum_{j \in[n]} c_{j} x_{i} & \\
\text { s.t. } & \sum_{j \in[n]}\left(\widehat{a}_{j}+\delta_{j} \Delta_{j}\right) x_{j} \leq b, & \\
& \sum_{j \in[n]} \delta_{j}=\Gamma, & j \in[n], \\
& \delta_{j} \in\{0,1\} & j \in[n] .
\end{array}
$$

Problem (9) is NP-hard, because the deterministic 0-1 knapsack problem, obtained by fixing $\Gamma=0$, is already NP-hard [6]. We will show that (9) can be reduced the following constrained shortest path problem in which: we are given a network $G=(V, A)$ with a $\operatorname{cost} c_{a} \geq 0$ and a weight $w_{a} \geq 0$ specified for each $\operatorname{arc} a \in A$. We seek a shortest $s-t$ path in $G$ whose total weight does not exceed $b$. This problem is NP-hard [6]. However, it can be solved in pseudopolynomial time $O(|A| b)$ in acyclic networks, assuming that $b \in \mathbb{Z}_{+}$, by using dynamic programming and it admits a fully polynomial approximation scheme (FPTAS) [7]. Consequently the problem (9) can be solved in pseudopolynomial time and has a FPTAS as well.

The method of constructing the corresponding network $G=(V, A)$ for $n=6$ and $\Gamma=4$ is shown in Fig. 3 (the idea for arbitrary $n$ and $\Gamma \in\{0, \ldots, n\}$ is the same). Network $G$ is composed of $n$ layers. In the $j$ th layer we consider all possible four cases for the variable $x_{j}$. Namely, $x_{j}=1$ and $\delta_{j}=0$ (solid horizontal arc); $x_{j}=0$ and $\delta_{j}=0$ (dashed horizontal arc); $x_{j}=1$ and $\delta_{j}=1$ (solid diagonal arc); $x_{j}=0$ and $\delta_{j}=1$ (dashed diagonal arc). We seek a longest $s-t$ path in $G$ whose weight is not greater than $b$. Observe that this problem can be easily reduced to the constrained shortest path problem by replacing $c_{j}$ with $c_{\max }-c_{j}$ for each $j \in[n]$, where $c_{\max }=\max _{j \in[n]} c_{j}$. Since each $s-t$ path has the same number of arcs, the longest constrained shortest path in $G$ is the same as the shortest constrained path in the modified network. 


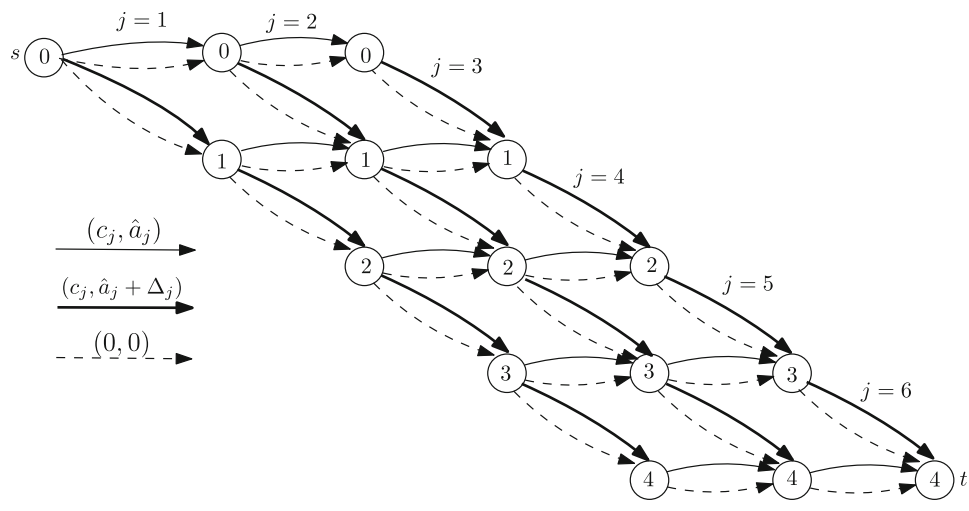

Fig. 3. Network for $n=6$ and $\Gamma=4$.

It is easy to see that each optimal solution to the constrained longest path problem in $G$ corresponds to an optimal solution to (9). Each feasible $s-t$ path in $G$ contains exactly $\Gamma$ diagonal arcs, which correspond to $\delta_{j}=1$, and exactly $n-\Gamma$ horizontal arcs which correspond to $\delta_{j}=0$. For each diagonal and horizontal arcs the path indicates whether $x_{j}=1$ or $x_{j}=0$, which provides a feasible solution to (9). Since the computed path is the longest one, it corresponds to an optimal solution to (9).

\subsection{Continuous 0-1 Knapsack Problem}

In this section we examine the continuous version of the 0-1 knapsack problem with uncertain weights, i.e. the model (7) discussed in Sect. 4 (a special case of problem (6)). This model can be linearized in a standard way by introducing additional $n$ variables $y_{j} \geq 0, j \in[n]$, which express $y_{j}=\delta_{j} x_{j}$, and $2 n$ constraints of the form $y_{j} \leq \delta_{j}, y_{j} \geq x_{j}-\left(1-\delta_{j}\right)$ for $j \in[n]$. In this section we will transform (7) into a mixed integer linear program having only $n+1$ variables (including $n$ binary variables) and at most $2 n+1$ additional linear constraints. We also propose an upper bound, which can be computed in $O\left(n^{2}\right)$ time.

Fix $\delta_{j}, j \in[n]$, and consider the following linear programming problem (with dual variables $\beta, \alpha_{j}$, in brackets):

$$
\begin{array}{lll}
\max & \sum_{j \in[n]} c_{j} x_{j} & \\
\text { s.t. } & \sum_{j \in[n]}\left(\widehat{a}_{j}+\delta_{j} \Delta_{j}\right) x_{j} \leq b \quad \\
& x_{j} \leq 1 & j \in[n],\left[\alpha_{j}\right], \\
& x_{j} \geq 0 & j \in[n] .
\end{array}
$$


The dual to $(10)$ is

$$
\begin{array}{ll}
\min & \beta b+\sum_{j \in[n]} \alpha_{j} \\
\text { s.t. } & \beta\left(\widehat{a}_{j}+\delta_{j} \Delta_{j}\right)+\alpha_{j} \geq c_{j} j \in[n], \\
& \alpha_{j} \geq 0 \\
& \beta \geq 0 .
\end{array}
$$

In an optimal solution to (11), we can fix $\alpha_{j}=\left[c_{j}-\beta\left(\widehat{a}_{j}+\delta_{j} \Delta_{j}\right)\right]_{+}$, where $[y]_{+}=\max \{0, y\}$. Hence $(11)$ can be rewritten as

$$
\min _{\beta \geq 0} g(\boldsymbol{\delta}, \beta)=\beta b+\sum_{j \in[n]}\left[c_{j}-\beta\left(\widehat{a}_{j}+\delta_{j} \Delta_{j}\right)\right]_{+} .
$$

Proposition 1. For any $\boldsymbol{\delta}$, the function $g(\boldsymbol{\delta}, \beta)$ attains minimum at $\beta=0$ or $\beta=\frac{c_{k}}{\widehat{a}_{k}}$ or $\beta_{k}=\frac{c_{k}}{\widehat{a}_{k}+\Delta_{k}}$ for some $k \in[n]$.

Proof. Let us reorder the variables so that

$$
\frac{c_{1}}{\widehat{a}_{1}+\delta_{1} \Delta_{1}} \geq \frac{c_{2}}{\widehat{a}_{2}+\delta_{2} \Delta_{2}} \geq \cdots \geq \frac{c_{n}}{\widehat{a}_{n}+\delta_{n} \Delta_{n}} .
$$

Let $k \in[n]$ be the smallest index in $[n]$ such that $\sum_{j \in[k]}\left(\widehat{a}_{j}+\delta_{j} \Delta_{j}\right)>b$. If there is no such $k$, then we fix $x_{j}=1$ for each $j \in[n]$, obtaining a feasible solution to (10) with the objective value $\sum_{j \in[n]} c_{j}$. The objective value of (11) for $\beta^{*}=0$ is also $\sum_{j \in[n]} c_{j}$ so, by strong duality $\beta^{*}=0$ is optimal.

Assume that $k<n$. Fix $b^{\prime}=\sum_{j \in[k-1]}\left(\widehat{a}_{j}+\delta_{j} \Delta_{j}\right) \leq b$ and $b^{\prime}=0$ if $k=1$. Let us construct a feasible solution to (10) by setting $x_{j}=1$ for $j \in[k-1]$ and $x_{k}=\left(b-b^{\prime}\right) /\left(\widehat{a}_{k}+\delta_{k} \Delta_{k}\right)$. The objective value of (10) is $\sum_{j \in[k-1]} c_{j}+c_{k} x_{k}$. Let us now construct a feasible solution to (11) by fixing $\beta^{*}=\frac{c_{k}}{\widehat{a}_{k}+\delta_{k} \Delta_{k}}$. Using the fact that the optimal $\alpha_{j}^{*}=\left[c_{j}-\beta\left(\widehat{a}_{j}+\delta_{j} \Delta_{j}\right)\right]_{+}, j \in[n]$, we conclude that $\alpha_{j}^{*}=0$ for $j \geq k$, and we get the objective value of (11)

$$
\frac{c_{k} b}{\widehat{a}_{k}+\delta_{k} \Delta_{k}}+\sum_{j \in[k-1]} c_{j}-\frac{c_{k}}{\widehat{a}_{k}+\delta_{k} \Delta_{k}} b^{\prime}=\sum_{j \in[k-1]} c_{j}+c_{k} x_{k} .
$$

Hence $\beta^{*}$ is optimal according to the strong duality. Since $\beta^{*}=\frac{c_{k}}{\widehat{a}_{k}+\delta_{k} \Delta_{k}}$ and $\delta_{k} \in\{0,1\}$ the proposition follows.

Using the fact that $\boldsymbol{\delta} \in\{0,1\}^{n}$, let us rewrite $g(\boldsymbol{\delta}, \beta)$ as follows:

$$
g(\boldsymbol{\delta}, \beta)=\beta b+\sum_{j \in[n]}\left(\left[c_{j}-\beta \widehat{a}_{j}\right]_{+}+\delta_{j}\left(\left[c_{j}-\beta\left(\widehat{a}_{j}+\Delta_{j}\right)\right]_{+}-\left[c_{j}-\beta \widehat{a}_{j}\right]_{+}\right) .\right.
$$

Setting $\phi(\beta)=\beta b+\sum_{j \in[n]}\left[c_{j}-\beta \widehat{a}_{j}\right]_{+}$and $\psi_{j}(\beta)=\left[c_{j}-\beta\left(\widehat{a}_{j}+\Delta_{j}\right)\right]_{+}-\left[c_{j}-\beta \widehat{a}_{j}\right]_{+}$ yields

$$
g(\boldsymbol{\delta}, \beta)=\phi(\beta)+\sum_{j \in[n]} \delta_{j} \psi_{j}(\beta)
$$


Fix $\mathcal{B}=\left\{\frac{c_{k}}{\widehat{a}_{k}}: k \in[n]\right\} \cup\left\{\frac{c_{k}}{\widehat{a}_{k}+\Delta_{k}}: k \in[n]\right\} \cup\{0\}$. Proposition 1 now shows that the optimal values of $\boldsymbol{\delta}$ can be found by solving the following max-min problem:

$$
\max _{\boldsymbol{\delta} \in \Phi} \min _{\beta \in \mathcal{B}} g(\boldsymbol{\delta}, \beta)
$$

which can be represented as the following program:

$$
\begin{array}{ll}
\max & t \\
\text { s.t. } & t \leq \phi(\beta)+\sum_{j \in[n]} \psi_{j}(\beta) \delta_{j} \beta \in \mathcal{B}, \\
& \sum_{j \in[n]} \delta_{j}=\Gamma, \\
& \delta_{j} \in\{0,1\} \quad j \in[n] .
\end{array}
$$

Model (14) has $n$ binary variables and one continuous variable. Since $|\mathcal{B}| \leq 2 n+1$, the number of constraints is at most $2 n+2$. Observe that the size of $\mathcal{B}$ can be smaller, since some ratios in $\mathcal{B}$ can be repeated. Unfortunately, no polynomial time algorithm for solving (14) is known, so the complexity of the problem remains open. Observe that we can use (13) to compute an upper bound for (10). Namely, by exchanging the min-max operators we get

$$
\max _{\boldsymbol{\delta} \in \Phi} \min _{\beta \in \mathcal{B}} g(\boldsymbol{\delta}, \beta) \leq \min _{\beta \in \mathcal{B}} \max _{\boldsymbol{\delta} \in \Phi} g(\boldsymbol{\delta}, \beta):=U B .
$$

For a fixed $\beta \in \mathcal{B}$, the optimal values of $\delta$ can be found in $O(n)$ time by solving a selection problem (see, e.g., [4]). Hence $U B$ can be computed in $O\left(n^{2}\right)$ time.

\section{Uncertain Right Hand Sides}

In this section we will show how to cope with uncertain right hand sides of the constraints. To simplify the presentation, we will assume that the constraint coefficients are deterministic. We thus study the following problem

$$
\begin{aligned}
\max z= & \boldsymbol{c}^{T} \boldsymbol{x} \\
& \boldsymbol{A} \boldsymbol{x} \leq \widetilde{\boldsymbol{b}}, \\
& \boldsymbol{x} \in \mathbb{X} .
\end{aligned}
$$

where $\boldsymbol{A}$ is $m \times n$ matrix of precise constraint coefficients and $\tilde{\boldsymbol{b}}$ is an $m$ vector of uncertain right hand sides. The meaning of $\mathbb{X}$ is the same is in the previous sections. Assume that $\tilde{b}_{i}$ is only known to belong to the interval $\left[\widehat{b}_{i}-\Delta_{i}, \widehat{b}_{i}+\Delta_{i}\right]$, $i \in[m]$. Let $\Phi=\left\{\boldsymbol{\delta}_{i} \in\{0,1\}^{m}: \sum_{i \in[m]} \delta_{i}=\Gamma\right\}$. A fixed vector $\boldsymbol{\delta} \in \Phi$ induces the uncertainty set $\mathcal{U}_{\boldsymbol{\delta}}=\left\{\boldsymbol{b} \in \mathbb{R}^{m}: b_{i} \in\left[\widehat{b}_{i}-\delta_{i} \Delta_{i}, \widehat{b}_{i}\right], i \in[m]\right\}$. Using the optimistic approach (see Sect. 2), we can transform (15) into the following problem:

$$
\begin{gathered}
\max z=\boldsymbol{c}^{T} \boldsymbol{x} \\
\min _{\boldsymbol{\delta} \in \Phi} \max _{\boldsymbol{b} \in \mathcal{U}_{\boldsymbol{\delta}}} \boldsymbol{A} \boldsymbol{x} \leq \boldsymbol{b}, \\
\boldsymbol{x} \in \mathbb{X} .
\end{gathered}
$$


Problem (16) can be rewritten as follows:

$$
\begin{aligned}
& \max \boldsymbol{c}^{T} \boldsymbol{x} \\
& \sum_{j \in[n]} \widehat{a}_{i j} x_{j} \leq \widehat{b}_{i}-\delta_{i} \Delta_{i} i \in[m], \\
& \sum_{i \in[m]} \delta_{i}=\Gamma \quad i \in[m], \\
& \delta_{i} \in\{0,1\} \quad i \in[m] . \\
& \boldsymbol{x} \in \mathbb{X} \text {. }
\end{aligned}
$$

Observe that (17) can be solved by trying all possible vectors of $\boldsymbol{\delta}$, which can be done in reasonable time if the number of constraints is not large. In particular, if $\mathbb{X}$ is a polyhedron and $m$ is constant, then (17) can be solved in polynomial time. The next theorem characterizes the problem complexity when $m$ is a part of input.

Theorem 2. Problem (17) is strongly NP-hard and not at all approximable even if $\mathbb{X}$ is a bounded polyhedron.

Proof. Consider the strongly NP-complete 3-SAT problem [6], in which we are given a set of boolean variables $\left\{x_{1}, \ldots, x_{n}\right\}$ and a set of clauses $C_{1}, \ldots, C_{m}$. Each clause $C_{i}$ contains three literals $\left\{p_{i}, q_{i}, r_{i}\right\}$, where $p_{i}, q_{i}, r_{i} \in\left\{x_{1}, \bar{x}_{1}, \ldots, x_{n}, \bar{x}_{n}\right\}$. We ask if there is a $0-1$ assignment to the variables which satisfies all the clauses. Given an instance of 3-SAT we build the following program:

$$
\begin{aligned}
& \max (1-t) \\
& -x_{j} \leq 0-\delta_{j} \cdot 1 \quad j \in[n], \\
& -\bar{x}_{j} \leq 0-\delta_{j}^{\prime} \cdot 1 \quad j \in[n], \\
& x_{j}+\bar{x}_{j} \leq 1 \quad j \in[n], \\
& \sum_{j \in[n]}\left(\delta_{j}+\delta_{j}^{\prime}\right)=n \text {, } \\
& p_{i}+q_{i}+r_{i} \geq 1-t \quad \forall C_{i}=\left\{p_{i}, q_{i}, r_{i}\right\} \text {, } \\
& \delta_{j}, \delta_{j}^{\prime} \in\{0,1\} \quad j \in[n], \\
& x_{j}, \bar{x}_{j} \in[0,1] \quad j \in[n] \text {, } \\
& t \in[0,1] \text {. }
\end{aligned}
$$

Notice that in any feasible solution to (18) we must have $\delta_{j}=1$ and $\delta_{j}^{\prime}=0$; or $\delta_{j}=0$ and $\delta_{j}^{\prime}=1$. Indeed, if $\delta_{j}=\delta_{j}^{\prime}=1$, then $x_{j} \geq 1, \bar{x}_{j} \geq 1$ which contradicts $x_{j}+\bar{x}_{j} \leq 1$. If $\delta_{j}=\delta_{j}^{\prime}=0$, then there must be some $k \in[n]$ such that $\delta_{k}=\delta_{k}^{\prime}=1$ and we again get contradiction. Now $\delta_{j}=1$ and $\delta_{j}^{\prime}=0$ implies $x_{j}=1$ and $\bar{x}_{j}=0$, and $\delta_{j}=0$ and $\delta_{j}^{\prime}=1$ implies $x_{j}=0$ and $\bar{x}_{j}=1$. The rest of the proof is the same as in the proof of Theorem 1.

\section{The Shortest Path Problem with Uncertain Costs}

Let $\mathbb{X}$ be the set of characteristic vectors of all $s-t$ paths in a given network $G=(V, A), \mathbb{X} \subseteq\{0,1\}^{|A|}$. Suppose that the arc costs are uncertain, and they 
are specified as intervals $\left[\widehat{c}_{i j}-\Delta_{i j}, \widehat{c}_{i j}+\Delta_{i j}\right],(i, j) \in A$. Applying the approach from Sect. 2, we get the following model:

$$
\begin{array}{ll}
\min & \sum_{(i, j) \in A}\left(c_{i j}+\delta_{i j} \Delta_{i j}\right) x_{i j} \\
\text { s.t } & \sum_{\substack{(i, j) \in A\\
}} \delta_{i j} \in\{0,1\} \quad(i, j) \in A, \\
& x \in \mathbb{X} .
\end{array}
$$

We now show that (19) can be solved in polynomial time. Given network $G=$ $(V, A)$, we form network $G^{\prime}=\left(V, A^{\prime}\right)$ having the same set of nodes with the same $s$ and $t$. For each arc $(i, j) \in A$ we create two parallel arcs, namely the solid $\operatorname{arc}(i, j) \in A^{\prime}$ with cost $\widehat{c}_{i j}$ and weight 1 and the dashed arc $(i, j) \in A^{\prime}$ with cost $\widehat{c}_{i j}+\Delta_{i j}$ and weight 0 . An example is shown in Fig. 4 . We solve the constrained shortest path problem in $G^{\prime}$ with $b=|A|-\Gamma$, i.e. we seek a shortest $s-t$ path in $G^{\prime}$ whose weight does not exceed $|A|-\Gamma$. This problem can be solved in $O(|A| b)$-time [7] which is $O\left(|A|^{2}\right)$, by the definition of $b$.
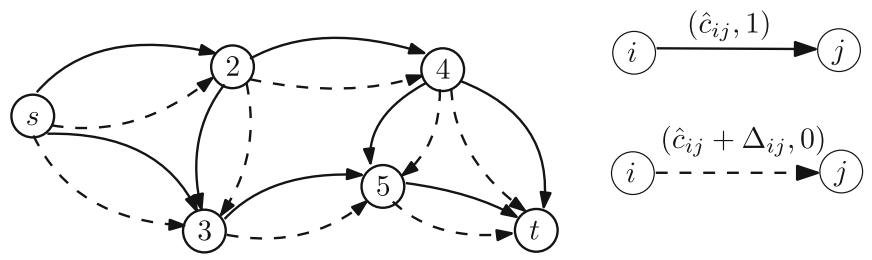

Fig. 4. Network $G^{\prime}$ for $G=(V, A)$. We seek a shortest $s-t$ path with weight at most $|A|-\Gamma$, i.e. which uses at most $|A|-\Gamma$ solid arcs.

To see that the transformation is correct, let $P$ be a path in $G^{\prime}$ with cost $c(P)$. Path $P$ is of the form $P_{S} \cup P_{D}$, where $P_{S}$ is the set of solid arcs in $P$ and $P_{D}$ is the set of dashed arcs is $P$. Since $\left|P_{S}\right| \leq|A|-\Gamma$, we get $\left|A \backslash P_{S}\right| \geq \Gamma$. We form a feasible solution to (19) with the cost at most $c(P)$ as follows. If $(i, j) \in P$ and $(i, j)$ is a solid arc, we fix $x_{i j}=1$ and $\delta_{i j}=0$; if $(i, j) \in P$ and $(i, j)$ is a dashed arc, we fix $x_{i j}=1$. We also fix $\delta_{i j}=1$ for any subset of $\Gamma \operatorname{arcs}$ in $A \backslash P_{S}$. It is easy to see that we get a feasible solution to (19) with the objective value at most $c(P)$. Conversely, let $\left(x_{i j}, \delta_{i j}\right)$ be a feasible solution to (19) with the objective value $c^{*}$. We construct a corresponding path $P$ in $G^{\prime}$ as follows. If $x_{i j}=1$ and $\delta_{i j}=0$, then we add the solid $\operatorname{arc}(i, j)$ to $P$; if $x_{i j}=1$ and $\delta_{i j}=1$, then we add the dashed arc $(i, j)$ to $P$. Since $x_{i j}$ describe an $s-t$ path in $G$, the set of $\operatorname{arcs} P$ is an $s-t$ path in $G^{\prime}$. Suppose that we chose more than $|A|-\Gamma$ solid arcs to construct $P$. Then $\left|\left\{(i, j) \in A: \delta_{i j}=0\right\}\right|>|A|-\Gamma$, and $\left|\left\{(i, j) \in A: \delta_{i j}=1\right\}\right|<\Gamma$, a contradiction with the feasibility of $\delta_{i j}$. Hence $P$ is a feasible path in $G^{\prime}$ and $c(P)$ is equal to $c^{*}$. So, the cost of an optimal path in $G^{\prime}$ is at most $c^{*}$. 
The proposed technique, consisting in arc duplication, can be used to solve other network problems. For example, when $\mathbb{X}$ is the set of characteristic vectors of spanning trees in $G$, then we can use an algorithm for the constrained spanning tree problem described in [11].

\section{Conclusion}

In this paper we have proposed a new approach to deal with uncertainty in optimization problems. Our idea is to soften the assumption that the worst scenario will occur for a given solution. We can thus use the pessimistic and the optimistic approaches to provide a broader family of solutions, one of which can be ultimately chosen by decision maker. Unfortunately, the proposed approach may lead to computationally harder problems. In particular, even the case of linear programming problems is NP-hard. However, we have shown in this paper some examples of optimization problems with uncertain parameters for which, after applying the approach, effective solution methods can be constructed.

The proposed approach can be too optimistic. Namely, the computed solution can be infeasible with large probability. Hence, and interesting research direction is to combine the pessimistic and the optimistic approaches, by using some aggregation methods.

\section{References}

1. Averbakh, I., Lebedev, V.: On the complexity of minmax regret linear programming. Eur. J. Oper. Res. 160, 227-231 (2005). https://doi.org/10.1016/j.ejor.2003. 07.007

2. Ben-Tal, A., El Ghaoui, L., Nemirovski, A.: Robust Optimization. Princeton Series in Applied Mathematics. Princeton University Press, Princeton (2009)

3. Bertsimas, D., Sim, M.: The price of robustness. Oper. Res. 52, 35-53 (2004). https://doi.org/10.1287/opre.1030.0065

4. Cormen, T., Leiserson, C., Rivest, R.: Introduction to Algorithms. MIT Press, Cambridge (1990)

5. Fischetti, M., Monaci, M.: Light robustness. In: Ahuja, R.K., Möhring, R.H., Zaroliagis, C.D. (eds.) Robust and Online Large-Scale Optimization. LNCS, vol. 5868, pp. 61-84. Springer, Heidelberg (2009). https://doi.org/10.1007/978-3-642-054655_3

6. Garey, M.R., Johnson, D.S.: Computers and Intractability. A Guide to the Theoryof NP-Completeness. W. H Freeman and Company, New York (1979)

7. Hassin, R.: Approximation schemes for the restricted shortest path problem. Math. Oper. Res. 17, 36-42 (1992). https://doi.org/10.1287/moor.17.1.36

8. Kall, P., Mayer, J.: Stochastic Linear Programming. Models, Theory, and Computation. Springer, Heidelberg (2011). https://doi.org/10.1007/978-1-44197729-8

9. Kasperski, A., Zieliński, P.: Robust discrete optimization under discrete and interval uncertainty: a survey. In: Doumpos, M., Zopounidis, C., Grigoroudis, E. (eds.) Robustness Analysis in Decision Aiding, Optimization, and Analytics. ISORMS, vol. 241, pp. 113-143. Springer, Cham (2016). https://doi.org/10.1007/978-3-31933121-8_6 
10. Kouvelis, P., Yu, G.: Robust Discrete Optimization and its Applications. Kluwer Academic Publishers, Boston (1997). https://doi.org/10.1007/978-1-4757-2620-6

11. Ravi, R., Goemans, M.X.: The constrained minimum spanning tree problem. In: Karlsson, R., Lingas, A. (eds.) SWAT 1996. LNCS, vol. 1097, pp. 66-75. Springer, Heidelberg (1996). https://doi.org/10.1007/3-540-61422-2_121

12. Schöbel, A.: Generalized light robustness and the trade-off between robustness and nominal quality. Math. Methods Oper. Res. 80(2), 161-191 (2014). https:// doi.org/10.1007/s00186-014-0474-9

13. Soyster, A.L.: Convex programming with set-inclusive constraints and applications to inexact linear programming. Oper. Res. 21, 1154-1157 (1973). https://doi.org/ 10.1287/opre.21.5.1154 